\title{
An Analysis of Artificial Intelligence Based Clinical Decision Support Systems
}

\author{
Schallig Matheus and Vaez Barzani Den \\ Biomedical Engineering, University of Groningen, Groningen, Netherlands. \\ matheusbio@aol.com
}

\begin{abstract}
Article Info
Journal of Biomedical and Sustainable Healthcare Applications (http://anapub.co.ke/journals/jbsha/jbsha.html) Doi: https://doi.org/10.53759/0088/JBSHA202101002
\end{abstract}

Received 15 August 2020; Revised form 18 September 2020; Accepted 22 October 2020.

Available online 05 January 2021.

(C)2021 Published by AnaPub Publications.

\begin{abstract}
The growing availability of medical data has sparked fresh interests in Computerized Clinical Decision Support Systems (CDSS), thanks to recent breakthroughs in machine and deep learning. CDSS has showed a lot of promise in terms of improving healthcare, enhancing the safety of patients and minimizing treatment costs. The application of CDSS, nonetheless, is unsafe since an insufficient or defective CDSS may possibly degrade healthcare quality and place patients at potential threat. Furthermore, the deployment of a CDSS may fail when the CDSS's output is ignored by its intended users owing to a lack of confidence, relevance, or actionability. We offer literature-based advice for the various elements of CDSS adoption, with a particular emphasis on Artificial Intelligence (AI) and Machine Learning (ML) systems: quality assurance, deployment, commissioning, acceptability tests, and selection, in this research. A critical selection process will assist in the process of identifying CDSS, which effectively suits the localized sites' needs and preferences. Acceptance testing ensures that the chosen CDSS meets the specified standards and meets the safety criteria. The CDSS will be ready for safe clinical usage at the local site once the commissioning procedure is completed. An efficient system implementation must result in a smooth rollout of the CDSS to well-trained end-users with reasonable expectations. Furthermore, quality assurance will ensure that the CDSS's levels are maintained and that any problems are discovered and resolved quickly. We conclude this research by discussing the methodical adoption process for CDSS to assist in avoiding issues, enhance the safety of patients and increasing quality of service.
\end{abstract}

Keywords - Computerized Clinical Decision Support Systems (CDSS), Machine Learning (ML), Artificial Intelligence (AI)

\section{INTRODUCTION}

The growing popularity of Machine Learning (ML) and Artificial Intelligence (AI), combined with the rising availability of medical studies, has generated an increase in popularity of AI applications in recent years, and Computerized Clinical Decision Support Systems (CDSS) in particular. A digital CDSS is defined in [1] as any software designed to aid physicians and consumers in medical decision. It is defined as "knowledge acquisition systems that use pieces of patient records to provide case-specific suggestions." Expert information and/or modeling developed using statistics and machine learning from data may be used by CDSS. CDSS were initially intended to gradually replace the health practitioners decision-making. A more sophisticated, modern view of the goal of CDSS is to assist the physician in achieving better decisions than either the physician or the CDSS can achieve on their own by evaluating the vast amount of available data. Contemporary CDSS often provide suggestions to physicians, and practitioners are urged to offer their individual decisions, rejecting CDSS advice that they believe are unsuitable.

Computerized CDSS have advanced significantly since their beginnings, including desktop treatment in MYCIN, HELP warning framework, and the Leeds Gastrointestinal Pain programs. The $5^{\text {th }}$ Integration with clinical processes and other clinical systems is one way they have progressed. They started off as stand-alone systems, requiring physicians to input patient data before viewing and interpreting the findings [2]. In 1967, CDSS were first integrated into medical information technologies, providing two key benefits: participants will not have to put data, and CDSS may be preemptive, alerting or proposing activities without the client proactively getting assistance from the CDSS. In the 1980s, the emergence and diffusion of protocols for describing, storing, and exchanging diagnostic data allowed the separation of knowledge base from CDSS application codes. Healthcare information systems began providing application programming interfaces (APIs) to connect with CDSS starting in 2005 [3], allowing for a more dynamic and less uniform interaction. CDSS have evolved into a broad range of kinds, which may be categorized based on a number of characteristics. As with alert systems, CDSS may provide assistance on demand or without prompting. CDSS may also be categorized depending on the technology that underpins them, such as principles, deep learning, reinforcement learning, evolutionary computation, or supervised learning, among other things.

CDSS enable diagnosis, outcome prediction, care plans, prescribing and managing medicines, preventive care, chronic illness monitoring, machine vision (contouring, recognition, and pathology detection), and a variety of other functions. Systematic studies show that using CDSS decreases unjustified practice variance, improves healthcare quality, eliminates wastes within the clinical systems, and minimizes the risks of medical practitioners being subjected to burnouts and 
overburden. Twenty to Twenty four years old CDSS, on the other hand, may have significant negative effects, since a defective CDSS or its improper usage might result in a decline in the quality of treatment. There are still a lot of ethical issues to be answered and patient safety concerns to be addressed. The traditional purpose of CDSS has been to support and advance clients (patients and clinicians) who are generally accustomed for medical decisions. Based on the recent advances in deep learning, CDSS are achieving human-level performance on a number of tasks, particularly image analysis, and are often functioning as "black boxes" with no explanation for their recommendations. This raises new issues about accountability and responsibilities. Regulatory procedures are changing in response, designating certain CDSS as clinical devices [4] (with official ramification) whereas eliminating others, e.g. the ones that skip the analysis of the images and allow clients to assess the recommendations' basis.

Even regulatory clearance, however, does not ensure a good outcome. CDSS may unintentionally increase physician burden. A well-known consequence of a CDSS notification system in healthcare management is "warning syndrome," which occurs when doctors learn to ignore cautions as a result of an overwhelming frequency of bogus alerts. Another threat of using CDSS would be that health professionals will end up losing their autonomy to make judgements or ascertain when it becomes suitable to supersede the Decision support system - as well as present breakthroughs in AI, which make that happen that CDSS could indeed make choices as well or better than living beings, make these consequences more pertinent. This may be useful in the case of a computerized system failure or the entrance of an individual with a very unusual health issue. As a consequence, it is essential to understand the positive and negative impacts of CDSS on medical decision. Although some CDSS were in operation for ages, their acceptance remains restricted due to a number of formulation and review issues, such as physicians' reluctance to use them leading to a shortage of time or confidence in the CDSS's outputs. Nonetheless, there is a significant potential need for CDSS due to the increasing quantity of available data, increasing diversity of medical interventions, and rapidly evolving medical technology.

CDSS may be useful for providing medical treatment that is customized to the preferences and biological features of patients. Patients may benefit from a pool of humanistic knowledge and professional experience that guides their diagnosis, therapy, and health monitoring. To enhance patient outcomes, minimize financial burden, and prevent unnecessary practice deviations, there is still a rising worldwide need for high-quality individual medicine. Machine learning-based CDSS are expected to help alleviate some of the current understanding and quality-of-care disparities across countries and localities. As a consequence, the problem of organizing, developing, delivering, executing, evaluating, and maintaining various decision support options for physicians, clients, and clients remains a hot subject in modern medicine. Section II focuses on the relevant literature assumptions regarding the aspect of external and internal factors influencing CDSS's adoption. Section III presents a research methodology. Section IV focuses on a critical evaluation of the research. Section V presents a brief discussion of the analysis while Section VI concludes the paper.

\section{LITERATURE REVIEW}

In [5], authors evaluated the factors beyond the control of the organization (external factors) influencing the adoption of CDSS. Lack of organizational capabilities to provide adequate engineering training and support was a major impediment, and it has been depicted to reduce users' trust in a framework and their way to fix any technological problems that may arise, deterring CDSS implementation. Culture is also identified by organizational theories as an enabler for facilitating the integration of any technological advances. Researchers discovered that young physicians were much more likely to need social commitment to embrace CDSS for antimicrobials administration, possibly since diagnostic and therapeutic leadership and senior healthcare professional preferences heavily influence young physicians' procedures, and seniors in our research have been less likely to embrace CDSS.

In [6], authors argue that the convenience of use is one of UTAUT's basic network quality constructs. In the study, physicians cited ease of use as a crucial component in their adoption and compliance to CDSS usages for antimicrobials administration. This is coherent with the information systems (IS) success model, which links users 'satisfaction and incorporation to ease of use. We discovered that one of the most significant characteristics for CDSS adoption is ease of use, according to general practitioners and those who have used it before. Because of the limited advisory time, workload, and risk of compromising direct relationships with patient populations due to the time needed to explore the system, ease of use is a major factor for CDSS execution in primary care.

In [7], authors argue that users' trust in the system's ability to meet their specific needs is related to system effort expectation and perceived benefit. End-user dialogue was rated as an essential coordinator for CDSS execution by healthcare professionals with more working experience, according to our findings. Similarly, clinicians with more nursing expertise (>10 years) were more probable in seeing CDSS as a risk to their clinical independence, according to our findings. As a result, including experienced physicians in the CDSS design and development phase is likely to result in improved system acceptability, trust, and adherence.

In [8], authors evaluated the internal constraints influencing the adoption and implementation of CDSS. Internal variables were consistently cited as obstacles to CDSS implementation for antimicrobials planning in their study, with mistrust in the system's composition being the most common. This was a frequent issue among CDSS nonusers in our research, which indicates that it may be the consequence of a lack of knowledge of how the system gathers data to make suggestions, as well as a lack of confidence in system developers and disagreement with the contents. Clinicians' concern that using CDSS might jeopardize individual clinical judgments enhances their reluctance to use the technology. Clinicians in our research who had used CDSS before were less likely to think that utilizing it would jeopardize their functional independence, demonstrating that end-user hesitancy to embrace CDSS for such reasons may be due to a misunderstanding 
of the technology rather than real experience with it. Experienced physicians were much less likely to utilize CDSS because they were afraid of jeopardizing established employment practices and losing autonomy over these procedures and the substance of therapeutic choices if they did. Younger doctors have greater technical competence and are much more competent in utilizing systems like CDSS, according to investigations.

In [9], authors argue that a greater percentage of younger physicians were CDSS users among our respondents than senior clinicians. A successful healthcare involvement approach with experienced physicians is needed to overcome obstacles to CDSS implementation. The goal would be to enable early adopters in this group to promote change and promote for CDSS usage among their peers. End-user opposition to CDSS implementation for antimicrobials administration may be influenced by clinicians' time restraints and the risk of workflow disruptions, according to our findings. This is in line with prior research that suggests that failing to offer a good match between appropriateness, format, and responsiveness of suggestions has a negative effect on CDSS adoption and use in general. Furthermore, our results show that time and workflow limitations are more likely to be seen as obstacles to CDSS implementation in primary care. Regardless of the fact that workflow interruptions and time sensitivity (high workloads) are common in all hospital environments, the requirement to evaluate clinical data in a brief consultation may contribute to CDSS deployment being restricted in primary care. In [10], researchers' results demonstrate how mediating variable factors like age, clinical experience, and ehealth literacy influence clinicians' behavior when it comes to implementing digital healthcare systems. These results are in line with previous research on the impact of these variables on the perceived utility of digital health systems and users' intentions to utilize them. Understanding users' propensity based on such mediator variables, for instance, it assist minimize variability in technologies adoption and allow a cultural change among all doctors. The focus of future research should be on developing recommendations and a regulatory framework to overcome the obstacles to CDSS implementation for antimicrobial administration.

The adoption and usage of various kinds of CDSS is influenced by a variety of person and contextual factors, according to our results. To assist CDSS adoption, further effort in addressing organizational obstacles and finding optimum frameworks in terms of preparation, administration, governance, and communications should be explored [11]. Because of the reduced participation rate, the outcomes may not be indicative of all Australian clinicians' experiences and attitudes. Although the response rate was lower than expected, it was comparable to other studies conducted with healthcare professionals. Selection bias may have been introduced as a result of participants' self-selection as a result of polarized opinions concerning the theme. Another demerit is the survey's sectional factor that makes it challenging to establish insightful causal conclusions. Respondents' views of CDSS variables for clinical treatment may differ from their actual practice. Some questions allowed for open answers, which may have helped to alleviate this to some degree. The goal of this article is to offer advice on the many phases of safely and effectively implementing CDSS (refer to Table I) in a clinic. Section III focuses on the methodology for the research.

\section{METHODOLOGY}

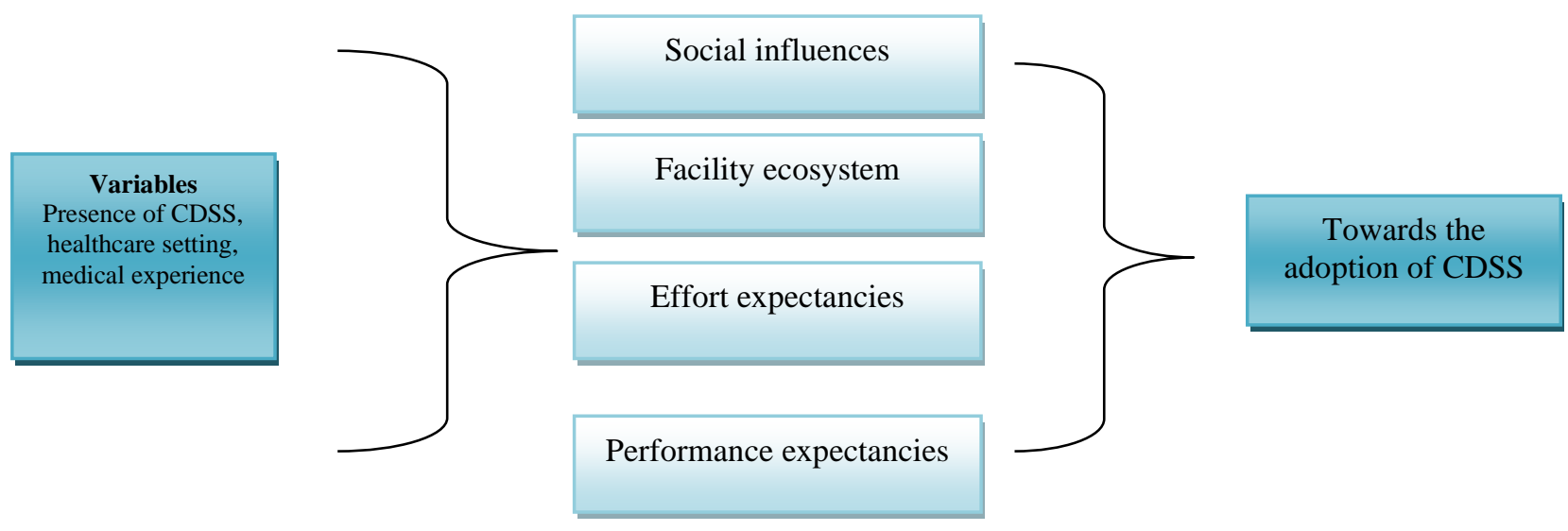

Fig 1. The theoretical foundation for the research, which is centered on the Unified theory

To investigate the interaction between several entities, technical metrics and humans influencing the adoption of CDSS and its application within the medical control system, we applied a Unified Theory of Acceptance and Use of Technology (UTAUT) paradigm. The bulk of computer methodologies provide comparable structures to explain acceptance and use of technology behavior. Researchers sought to choose the most practical aspects from these models, limiting the framework's total breadth and depth. To address this, the UTAUT included eight of the most widely used models to provide a complete framework for behavioural intent to embrace and utilize technology. The four major components that affect technology acceptance in the UTAUT model are effort expectation, relative advantage, cultural pressure, and the enabling environment. The integrity of the software architecture in terms of simplicity of use, incorporation into a regular workflow, and perceived advantages of increasing performance are linked to effort and output expectation. The impact of social connections in an organization on users' behavior to embrace and utilize any technology is referred to as social pressure. The users' perception that any location or organization has a suitable system in place to maintain usage of the technology is 
captured by the program's last component, the enabling environment. Although the environment has no bearing on the users' goals, it does have a direct effect on technology acceptance and utilization. Integration of user-specific variables that limit the effect of model components is one of the most important features of UTAUT. The direction and degree of the impact of model components on behaviour intention and practical use of technologies are influenced by these moderating factors, which include age, ethnicity, and expertise.

\section{CRITICAL ANALYSIS}

\section{Selection}

In recent times, the number of commercially accessible CDSS for medical applications has increased. As a result, choosing the most suitable CDSS among the many options that are provided is not always simple, but it is an important step in implementing an effective CDSS.

Table 1. An overview of the steps required in implementing a CDSS

\begin{tabular}{lll}
\hline \multicolumn{1}{c}{ Phases } & \multicolumn{1}{c}{ Objectives } \\
\hline 1. Selections & $\begin{array}{l}\text { Selecting the best CDSS based on the match available with } \\
\text { the projected application and medical workflows, rights, } \\
\text { acceptability and productivity. } \\
\text { Tests, which CDSS satisfies the aspects of safety, privacy, } \\
\text { security needs applied for medical devices, handling normal } \\
\text { error cases, unforesen factors and exception conditions. }\end{array}$ \\
2. Acceptance tests & $\begin{array}{l}\text { Preparing CDSS for more optimized applications of the } \\
\text { clinical tests based on performance and safety within the } \\
\text { localized contexts. } \\
\text { Rolling out CDSS and the changes from the ancient } \\
\text { workflows to the novel after training the end user and } \\
\text { effectively managing expectations. } \\
\text { Making sure that quality provision of CDSS is enough for } \\
\text { tracking both the external and internal updates and the } \\
\text { contextual changes. }\end{array}$ \\
4. Implementations &
\end{tabular}

CDSS user acceptability is important; many implementation studies indicate that doctors' and other healthcare professionals' perceptions of how helpful a CDSS is affects its adoption and use. Resultantly the first phase in the development should be to establish a multidisciplinary advisory panel largely composed of main diagnostic investors and shareholders, or by a amount of specialist doctor "champs," physician authorities, divisional superintendents, and system analysts, who really are able to make better decisions and be ready to take responsibility for the enlistment of a CDSS. Including end users in CDSS deployment rather than forcefully imposing the CDSS on them improves the probability of user adoption. To be effective, the CDSS should be conceived as component of a broader, more coherent, and division quality improvement strategy, with a therapeutic quality gap clearly established and assessed between current outcomes for patients or procedures and the desired eventual outcome. When choosing a CDSS, the accuracy of the CDSS or how it fits inside the clinical performance gap are the two most important factors to consider. The integrity of a CDSS must be assessed on at least two variables: the technological infrastructure and the data or expertise that was utilized to create it. CDSS should be developed, developed, tested, and reported using widely accepted quality assurance techniques for computer programming in the medical sector, since it is software that may be used as a medical product. The CDSS's medical science cannot be proved to be clinically comprehensive or technically accurate, but it must try to represent the present degree of career and scientific thought. Additionally, the necessary medical knowledge must be officially verified to meet specific criteria, such as being impartial, consistently understood, and properly finished.

A quality evaluation of the sourcedata is required when employing CDSS computer models learned via statistical analysis or machine learning. "Because the "garbage in" garbage out" concept applies to ML in particular, data quality is critical. High-quality data is described as having a comprehensive, undisturbed estimate of the area (patient or health manifestations) being modeled, and more precisely, as having a reflective, undisturbed estimate of the area (patients or health manifestations) being formed. Detection, data cleaning, and incomplete or absent data handling procedures should be collected, the data, and possible biases should have been evaluated and rectified [12]. The effectiveness of a CDSS is a significant indication of its quality. Various kinds of CDSS have different performance metrics. The area underneath the receiver operating characteristics (ROCs) curve, for instance, are frequently employed performance metrics in CDSS conducting outcome prediction. Performance may also be evaluated in terms of time saved in certain situations. However, evaluating success may be difficult, particularly when there is no gold standard of performance, as in the instance of therapy systems, where even specialists might differ.

Finally, the impact of the CDSS on healthcare outcomes or procedures is the most complex to quantify but most important performance indicator. The publication of comprehensive assessments of the accessibility and efficacy of CDSS deployment by CDSS suppliers may aid buying choices, however it should be noted that trials performed by CDSS programmers may exaggerate its advantages, necessitating third-party external verification. A comprehensive hazard analysis, which results in an extensive list of potential hazards and their potential effects, as well as a risk mitigation 
strategy, is part of the legislative framework and may offer significant insight into the CDSS's acceptability. The desirability of the CDSS must be evaluated and balanced against performance throughout the selection process. The robustness of the data proving medical suggestions given by CDSS should be visible to users in order for them to trust the CDSS's result. Models predicated on hand-engineered parameters and basic algorithms ( - for example, decision trees) have greater degrees of concreteness or interpretability than any of those dependent on more sophisticated methods like random forest and supervised learning. As previously said, selecting a CDSS that meets the needs of the localized site is critical.

The selection procedure has to be restricted to CDSS, which accesses the required populations, assess the integrated converters and interventions, and focus on the projected results, according to the PICO framework. When choosing a CDSS, keep the five "rights" in mind: providing the factual data (what), to an appropriate person (who), in the correct format (how), through the appropriate channels (in which), and at the correct time in the process (when). The CDSS's outputs (clinical suggestions and evaluations) ought to be clinically meaningful, short, clear, and effective in order to provide the proper data. The CDSS should also be linked as tightly as possible into its users' current workflow, for instance, into the HER, minimizing the efforts required by clients to obtain or react on systems suggestions. Modification of a CDSS may be required in order for it to suit the workflows of a certain clinic. As a result, the customization capabilities of each CDSS should be considered while making a decision. Another factor to examine in the localized workflows is if the various datasets needed for CDSS to operate properly is accessible at that point in the process. Another thing to think about while choosing a CDSS is its usability. More precisely, how simple is it to utilize the CDSS, as well as how much treatment is required? Vendors must be upfront about the level of skill needed to operate the system. When deciding on a CDSS, cost-effectiveness ought to be a key factor to examine, especially in comparison to other CDSS or healthcare technologies (e.g., a new piece of equipment) [13]. Nevertheless, demonstrating the CDSS's return on investment is challenging, particularly given the numerous conflicting objectives at the delivery mechanism levels.

Prior to purchasing a CDSS, a thorough analysis of the expenses associated with its procurement should be conducted, including one-time expenditures (buying, training, installation, etc.) as well as ongoing costs, eg the usage of resources and maintainability (such as users' time). The have to be assessed based on not just projected health improvements, and also projected savings through CDSS-facilitated efficiency. The interoperability of older initiatives, the maturation of the CDSS, as well as the availability of updates are all variables to consider.

\section{Acceptance Testing}

CDSS can effectively be visualized as a clinical tool that enhances acceptability tests with various processes in plan amongst medical practitioners. Acceptance criteria for medical equipment ensure that all of the device's stated parameters are met, as well as ensuring it meets all relevant safety standards. These tests are typically specified by the CDSS manufacturer; however they should be accompanied by the formation of local site personnel. The acceptability statement will be approved and the payments for the devices will be authorized after the satisfactory implementation of the acceptance criteria. As a result, the collection of test scenarios must be extensive, including situations that fall beyond the CDSS's domain, known as corner cases. Acceptance testing should be performed by technological advocates for technical elements, while usability and specific clinical assessments should be performed by a subset of users who represent a random group of the target end-user demographic for usability and specific clinical tests. At the very least, the acceptability test plan should include the following elements:

- The device's design and implementation

- $\quad$ APIs provided by the CDSS are working properly (if any)

- A comprehensive tour of the interface, including how to utilize the CDSS as part of an existing process

- The outputs of the CDSS should be clinically comprehensive, relevant, comprehensible, consistent, and repeatable

- $\quad$ Functions of auditing, security, and privacy

- Unexpected, inaccurate, or inadequate input data, sudden closure situations (for example, owing to a power loss), which result in unfinished operations, and so on are common error circumstances.

In the case of inadequate or inaccurate data, the CDSS must not make incorrect suggestions. Furthermore, the CDSS is required to manage these circumstances by maintaining internal consistency, sending appropriate error signals, and, if necessary, shutting down in a controlled manner. In response to the above, CDSS testing phase should ensure that the CDSS recommendations are accurate. Patients' safety and well-being may be jeopardized if suggestions are inaccurate. These assessments must contrast the CDSS's results to the anticipated results of a fixed, limited, but random sample of actual instances. The predicted reliability based on the acceptance criteria findings should be checked to the vendor's stated efficiency and statistically evaluated to see whether it falls within the error tolerance. The same may be said for the vendor's other quantity and quality estimations. To compute the likelihood that the effectiveness evaluated in the samples is similar to the probabilistic density element illustrated by the accuracies and error-tolerant tool, such as Mann Whitney U-tests) should be applied. If the estimated likelihood is less than a particular level of significance, we can rule out the possibility that the absolute precision is within the confidence interval. Lastly, as part of acceptability testing, verification for validity and usability of the CDSS handbook would be essential for beginners or in emergencies or exceptional circumstances. 


\section{Commissioning}

The procedure of licensing qualifies the CDSS for secure medical usage in the localized area, ensuring that it meets defined specifications and finished requirements. As a result, licensing confirms that the CDSS was implemented at the localized facility in accordance with the agreed-upon specifications, was efficiently turned over by the supplier, and, most important, that it operates effectively. It is commonly recommended that you prepare for this process by creating a contracting strategy that outlines the activities, routine, and obligated sentient and machinery assets, as well as the budget for this process needed from the help of the CDSS vendors. The application of the local site is the initial phase in the process of commissioning that, in the case of CDSS, eventually necessitates some modification or customizing. Customizing may be necessary for technological or security concerns, such as ensuring that CDSS variables are properly connected to the native EHR and that the CDSS and localized EHR interpretations of medical words are in sync.

Customizing is another effective approach for making the CDSS outputs more meaningful, valuable, and secure to use. According to a quantitative analysis in [14], all outstanding organizations spent a significant amount of time customizing their CDSS. To reduce warning tiredness, one form of customisation may be to analyze and enhance the relevance of warnings. A test strategy must be established and conducted to ensure that the deployed CDSS performs effectively in the localized location. To begin, the CDSS installation will almost certainly necessitate some adjustments in user routine. In that situation, the data required to sustain the upcoming workflows must be defined, and the proposed procedure must be thoroughly verified. Once the revised procedure is in place, the goal is to verify as many medically important situations and extreme situations as feasible to confirm that the CDSS is working effectively. The advisory board, which should be composed of doctors, executives, and informational technology professionals, should be engaged in recognizing all important circumstances and occurrences where the deployed CDSS could fail in the localized sites context, resulting in low performance or stability.

If a dataset with prior instances exists, a collection of previous cases that comprises challenges and unique instances as well as a reasonable sampling of the selected case populations could be retroactively examined. In this situation, the CDSS's suggestions are either examined by a committee of medical professionals in a blind test in which the specialists disregard the CDSS's outputs or compared to earlier judgments. CDSS should, nevertheless, be validated on genuine instances from the participants' actual medical practices before being implemented. One alternative is to examine the CDSS prospective by implementing a test operation in which the CDSS is utilized in tandem with the current process or supervised, with the present process serving as a backup. Random sampling, inputs selections, and sequence assessment are some of the methods used to cover a representative group of common and uncommon scenarios. During the pilots, it would be useful to examine the CDSS' medical significance in respect of customer acceptability, compliance to the CDSS suggestions, and responses to different choices and, eventually, the patient's medical results. Substantial differences in the CDSS's anticipated performances during this stage versus acceptability tests or the company's promises of efficiency and errors tolerance should be reviewed with the vendor. Criticality analytics is an essential aspect of commission tests because it simulates information input errors and analyzes and tests the behaviour of the CDSS for reliability. Validation is particularly vital during commissions to provide regional clinicians assurance that the supporting systems will work in their settings [16-17].

\section{Implementation}

The effectiveness of a CDSS depends heavily on the deployment procedure. This includes the designing and implementation of the distribution strategy, as well as the transfer from the initial to the current workflow that integrates CDSS and the application of CDSS inside the localized website. A successful CDSS installation necessitates equipping both consumers and the localized platform's infrastructures for widely spread CDSS use. Technical preparations will differ between CDSSs and localized websites, but there are certain general characteristics in how to educate consumers for the application of novel CDSS. It is critical to interact with and teach the affected customers before and during deployment. Proper learning of all partners and targeted consumers of the CDSS is critical to its effectiveness, and should include topics such as when to be used, how to apply it, the manner in which CDSS output can be comprehended, the time overruling CDSS recommendations, etc. This also integrates aiding individuals to understand the manner in which CDSS will affect their daily operations as well as how they can submit responses. It's critical to control customers' expectations regarding efficacy and accuracy as part of training, and to ensure that participants understand the CDSS's capabilities and limits. Stakeholder may have varying aspirations: Some regard CDSS as mainly a tool for improving uniformity, quality, and safety, whereas doctors may have a different perspective. Consumers should expect to take a step of belief during instruction: a CDSS will only be used if those who consume it genuinely think it is beneficial, but the benefits of the CDSS will only be realized after overcoming the initial complications. Hands-on training, as well as on-site provider from providers as needed to assist with just about any imperative complications that may arise, is a helpful resource because consumers may demand some assistance at first. The CDSS may be deployed or rolled out in stages (for example, spreading it out at a single comment or station to "iron out the bugs") or at once, which needs careful planning.

Quality Assurance (QA)

Prior to the CDSS's installation, a good manufacturing practices and strategy must be developed to guarantee that the CDSS's effectiveness and reliability are maintained by guaranteeing that its integrity stays appropriate for the function during its lifetime. Performance must be established as component of a CDSS's Quality assurance program employing just a few production metrics so the CDSS's impact can be tracked over period. Patient safety and changing patient outcomes 
are examples of performance indicators that are either specific to the CDSS's operation, such as high sensitivity and specificity for a clinical instrument or precision and accuracy for a diagnostic and therapeutic instrument, such as life expectancy. Savings, such as expenditures and productivity, may be used to determine efficiency. It is particularly important to evaluate baseline benchmarks, i.e., before to the deployment of a CDSS, and establish an approximation of the goal efficiency beforehand in order to examine the CDSS effectiveness. As per the QA approach, any flaws must be identified and corrected as soon as possible. To make it simpler to identify CDSS problems, mechanisms for collecting users' responses and performing it should be in place. Furthermore, CDSS flaws can be detected using a combination of qualitative and quantitative analysis, such as alert program firingrates or likely dominates for the recommenders CDSS. The visual identification and statistical procedure control evaluate have both proved to be useful techniques for identifying malfunction.

Aside from glitches, it's essential to register or record occurrences within which the CDSS has been contravened, as in when a notification has been neglected or a referral was overlooked, but since recognizing number of times and also why the CDSS is resisted could provide additional insight and contribute to the understanding of hitherto unrecognized imperfections. Correspondingly, keeping track of how well the CDSS is being utilized is important, since this may contribute to poor performance. To guarantee the integrity of input data, local sites must develop and execute internal data quality standards. CDSS data suppliers should be taught the significant of quality information and data and their applicability for enhancing the aspect of accuracy. According to the QA strategy, the CDSS's performance and safety must be maintained over time. In this way, the a CDSS is similar to the diagnosis plan framework or the therapy linear accelerators in that deviations from specific tolerance limits can lead to medical malpractices. These required an updated forecast framework with present dataset collecting in order to retain the effectiveness of their RT-induced harmful estimating methods. Contextual (ecological) drifting over times is the primary issue in this area. Adjustments in medical care can be severe, such as the emergence of graphics radiation treatment or surgical robots, or iterative, such as the population of participants with oropharynx villous cell conveying the p16 nutrients from sentient papillomaviral infestation.

Modifications in patient's condition mix, medication and therapy obsolescence, and recompiling of prognostic-clinical characteristics and therapeutic standards due to new randomised studies may all lead to an unfavorable dispersion in CDSS prescriptions over times. During CDSS acceptance testing and commissioning, such changes are difficult to anticipate, and they may result in time-dependent inconsistencies that contradict the CDSS's original assumptions. These transitions could be credited to a CDSS's insight, like individual medical demonstration that has changed considerably because the CDSS was commissioned, disclosing a heretofore unknown systematic error into certain clinical populations. Inner (modeling) drifting would be another factor that may cause chronological dispersion. Even after the original CDSS has been decommissioned, the models that underpin the CDSS are expected to be updated at regular intervals. Furthermore, models built with small sample sizes may exhibit some systematic bias at initially, but this will diminish over time as the models are trained and verified on larger datasets [15]. One of the following techniques could be used to refresh models: (a) adjusting the benchmark overall risk as well as (for boolean modeling techniques) the lower limit benefit for secondary endpoints, (b) calculating positive numbers for an established range of constraints, or (c) retooling the prototype on updated information, which may result in additional design variables, multipliers, and (for secondary endpoints) new thresholds.

Maintaining and evaluating incident continuous monitoring for improper or inaccurate CDSS reactions may help to prevent interior and exterior deviation. Concurrently, a "repetitive local verification" population must be set up on occasion, or best still, on a regular schedule, to properly evaluate various tests performed during activation. The recurrence may assist guarantee that the CDSS stays clinically relevant even if local force or evidence-based prescriptions alter. When delivering a CDSS upgrade, having a continuously local verification architecture is advantageous. Ultimately, while no CDSS could ever be flawless, the quality management system would verify which the CDSS's functionality satisfies standards established by the commission as a preliminary step. Establishing an updated monitoring system would be among the major objectives for the CDSS advisory board. Like some other electronic health records, CDSS will almost likely be upgraded "remotely." A CDSS is recently suspended from therapeutic applications and put in "service" service as a result of a vendor's- or consumer modification requests. During the servicing phase, further modifications are made, such as installing a software program upgrade or fixing a broken functionality. Having followed any upgrade, "diagnostic manual," or acknowledgement of the service back into medically operating condition, should only be permitted after predefined CDSS efficiency confirmation experiments have indeed been conducted on the system described, as with other areas of healthcare system upgrades and quality management. The requisite procedures should be specified by the manufacturer or the management handbook depending on the security evaluations; however it may be prudent to include some additional checks from customer acceptability techniques to ensure that all of CDSS's core operation has already been reinstalled after the update.

\section{DISCUSSION}

Clinical decision support systems (CDSSs) are ehealth systems that provide data to physicians at the point - of - care. These technologies filter and display reliable, factual evidence to aid medical decision by integrating evidence-based knowledge on optimal antimicrobial therapy with health information. The advantages of using CDSS for medication safety include improving the prescriptions procedure by evaluating decisions and deliver direct feedback, as well as boosting conformity with physician practice regulations and lowering the risk of superfluous and inadequate prescribing practices. Although there is conflicting data on the effectiveness of CDSS for antibiotics administration, some studies have indicated 
that it may reduce antibiotic treatment duration, length of hospitalisation, antibiotics treatment costs, and in-hospital fatality. End-user adoption of CDSS is not guaranteed by its availability, according to studies. Resultantly, irrespective of the significant evidence of the merits of CDSS, the involvement of end-users with the system is significantly minima. CDSS deployment is lower than expected in healthcare organizations, with 96 percent of CDSS notifications or suggestions being overruled or neglected for factors contributed to end-users' negative attitudes, evasion, or skepticism toward the system, and the system's unexpected effects on clinical practices.

Clinical culture, procedures, protocols, including ethical guidelines all play a role in the effective implementation of systems like CDSS in the healthcare setting. The intricate interplay of system, organizational, and human variables may make CDSS deployment difficult. Because of this complication, it's difficult to guarantee that improving one element of the patient care doesn't have unintended effects in another. The CDSS is particularly difficult to deploy since its reach goes much beyond that of a conventional information technology tool, incorporating an evidence-based perspective into everyday clinical practice. The proven findings in the network may at times contravene strongly held beliefs about medical impartiality and authority inequalities in the clinical context, contributing to skepticism regarding CDSS use. Many research have focused on technological suitability and user experience to better understand variables influencing CDSS acceptance, but little is known about how end-users' individual qualities affect views of CDSS adoption for antibiotic control.

CDSS upgrade "internet" should be addressed with medical systems moving to "cloud computing," increasing system automation, and hypothetical techniques which may adapt "on fly." A CDSS of this type could be permitted to evolve in actual time based on user engagement and its recommendations, with the CDSS' tendencies altering marginally with each involvement. The update administration protocol may allow for online updates, presenting a new and intriguing issue of balancing the possibility for ongoing improved performance against the danger of undiscovered performance deterioration owing to cognitive distortions in input, for example. A regular QA test program that defines which checks should be performed, while they should be performed, and by whom should be implemented as a high priority. Various elements of the CDSS's functioning are evaluated against a pre-determined ground truth as part of the QA process. The kinds of QA activities are often taken from the same tests that are used during commissioning. As a consequence, the recorded commissioning findings may be repeated at predetermined time intervals to ensure, which the CDSS function is not diminished over a significant duration of time. Several mathematical modelling studies have been presented and reviewed in the research when it comes to outlier recognition on CDSS over time, and the best technique will rely on the CDSS's nature.

The kind and frequency of these QA tests are determined by the probability of an unfavorable variation in CDSS functionality and the possible repercussions. For very probable failures or non-conformance events with significant implications, QA tests should be conducted more often. Unlikely failures and occurrences with little clinical implications should only be evaluated on a periodic basis. Because uncommon failures with serious implications may not be readily detected inside a regular QA program, a significant amount of work should be devoted into operational mitigation. It is suggested that the responsible employees have or learn a set of statistical and methodological approach via training in order to properly develop and implement the QA strategy. Medical scientists will be able to detect and explain faults in the CDSS if they have a thorough understanding as to how the underlying system functions. This training may be provided by the vendor or by third-party training providers. AI and ML will potentially be included in therapeutic physics classes, allowing for a more comprehensive knowledge of such technologies.

\section{CONCLUSION}

CDSS has showed tremendous promise in terms of enhancing medical and quality care while also decreasing unjustified variance, resource usage, and costs. AI-based CDSS have lately made a name for themselves by leveraging the growing accessibility of diagnostic data to help patients and clinicians in different setting (such as by providing more tailored projection of medical results or recommending treatment and diagnosis based on the application of EHRs, and massive data such as clinical imaging). A CDSS that is incorrect or ineffective, on the other hand, may degrade healthcare quality and place consumers at danger. Clustering, bias, and limits in the data for training the AI are some of the other risks associated with AI-based CDSS. These factors may cause the CDSS to be unable to generalization from training examples, resulting in undetected underperformance at the local location. As a result, care is required in order to mitigate CDSS's possible harmful effects. It is critical to choose a CDSS that is compatible with the local site's needs. CDSS, like any other medical equipment, requires rigorous testing process, installation, and quality control on the part of the local site. Furthermore, overcoming obstacles to an effective CDSS requires an effective implementation strategy. We collected the material from the study in this assessment to give CDSS programmers guidance. We conclude that adopting a systematic perspective to the many aspects of CDSS implementation will help to minimize mistakes, improve patient security, and increase the likelihood of success.

\section{References}

[1]. M. Tavakoli, H. Davies and R. Thomson, "Decision analysis in evidence-based decision making", Journal of Evaluation in Clinical Practice, vol. 6, no. 2, pp. 111-120, 2000. Doi : 10.1046/j.1365-2753.2000.00233.x.

[2]. A. A and P. P, "Evidence Based Medicine: Key Aspects in Clinical Decision Making", Scholars Academic Journal of Pharmacy, vol. 5, no. 7, pp. 262-267, 2016. Doi : 10.21276/sajp.2016.5.7.1.

[3]. A. Cohen, "Clinical Decision Making in an Era of DRG-Based Prospective Payment", Medical Decision Making, vol. 5, no. 1, pp. 3-6, 1985. Doi : 10.1177/0272989x8500500101.

[4]. S. Williams, "The Impact of DRG-Based Prospective Payment on Clinical Decision Making", Medical Decision Making, vol. 5, no. 1, pp. 2329, 1985. Doi : 10.1177/0272989x8500500106. 
[5]. T. Tulabandhula, "Interactions between learning and decision making", AI Matters, vol. 3, no. 1, pp. 25-26, 2017. Doi : $10.1145 / 3067682.3067691$

[6]. S. Holm, "Handle with care: Assessing performance measures of medical AI for shared clinical decision-making", Bioethics, 2021. Doi : 10.1111/bioe.12930.

[7]. P. Falzer and M. Garman, "A conditional model of evidence-based decision making", Journal of Evaluation in Clinical Practice, vol. 15, no. 6, pp. 1142-1151, 2009. Doi : 10.1111/j.1365-2753.2009.01315.x.

[8]. V. Torra, Y. Narukawa, J. Yin and J. Long, "Technologies for Decision Making and AI Applications", International Journal of Intelligent Systems, vol. 28, no. 6, pp. 523-523, 2013. Doi : 10.1002/int.21590.

[9]. A. Haldorai and A. Ramu, "Canonical Correlation Analysis Based Hyper Basis Feedforward Neural Network Classification for Urban Sustainability," Neural Processing Letters, Aug. 2020. doi:10.1007/s11063-020-10327-3

[10]. D. Devikanniga, A. Ramu, and A. Haldorai, "Efficient Diagnosis of Liver Disease using Support Vector Machine Optimized with Crows Search Algorithm," EAI Endorsed Transactions on Energy Web, p. 164177, Jul. 2018. doi:10.4108/eai.13-7-2018.164177

[11]. L. De Panfilis, C. Peruselli, S. Tanzi and C. Botrugno, "AI-based clinical decision-making systems in palliative medicine: ethical challenges", BMJ Supportive \& Palliative Care, pp. bmjspcare-2021-002948, 2021. Doi : 10.1136/bmjspcare-2021-002948.

[12]. T. Noseworthy and M. Watanabe, "Health policy directions for evidence-based decision making in Canada", Journal of Evaluation in Clinical Practice, vol. 5, no. 2, pp. 227-242, 1999. Doi : 10.1046/j.1365-2753.1999.00198.x.

[13]. E. Nouri, K. Georgila and D. Traum, "Culture-specific models of negotiation for virtual characters: multi-attribute decision-making based on culture-specific values", AI \& SOCIETY, vol. 32, no. 1, pp. 51-63, 2014. Doi : 10.1007/s00146-014-0570-7.

[14]. A. Islam and K. Chang, "Real-Time AI-Based Informational Decision-Making Support System Utilizing Dynamic Text Sources", Applied Sciences, vol. 11, no. 13, p. 6237, 2021. Doi : 10.3390/app11136237.

[15]. A. Gillies and P. Smith, "Can AI systems meet the ethical requirements of professional decision-making in health care?", AI and Ethics, 2021 Doi : 10.1007/s43681-021-00085-w.

[16]. A. Zia and C. Koliba, "The emergence of attractors under multi-level institutional designs: agent-based modeling of intergovernmental decision making for funding transportation projects", AI \& SOCIETY, vol. 30, no. 3, pp. 315-331, 2013. Doi : 10.1007/s00146-013-0527-2.

[17]. D. Partridge, "Human decision making \& the symbolic search space paradigm in AI", AI \& Society, vol. 1, no. 2, pp. 103-114, 1987. Doi : $10.1007 / \mathrm{bf} 01891271$. 